\title{
Parâmetros psicométricos das Escalas Florence de Cyber Agressão - Cyber Vitimização
}

\author{
Jaqueline Gomes Cavalcanti ${ }^{1}$ \\ Tamyres Tomaz Paiva ${ }^{2}$ \\ Carlos Eduardo Pimentel ${ }^{3}$ \\ Adriele Vieira de Lima Pinto ${ }^{4}$ \\ Giovanna Barroca de Moura $^{5}$
}

${ }^{1}$ Instituto de Educação Superior da Paraíba, PB, Brasil

https://orcid.org/0000-0002-3068-404X

${ }^{2}$ Universidade Federal da Paraiba, PB, Brasil

https://orcid.org/0000-0001-9415-0963

${ }^{3}$ Universidade Federal da Paraíba, PB, Brasil

https://orcid.org/0000-0003-3894-5790

${ }^{4}$ Universidade Federal da Paraíba, PB, Brasil

https://orcid.org/0000-0003-4126-1795

${ }^{5}$ Universidade Estadual do Vale do Acaraú, PB, Brasil -

https://orcid.org/0000-0001-7970-4323

\begin{abstract}
Resumo
O presente estudo objetivou adaptar a Escala Florence de Cyber Agressão - Cyber Vitimização (FCBVSs) para o contexto brasileiro, reunindo evidências de validade (fatorial e convergente); bem como de consistência interna. Para isso, contou-se com uma amostra de 315 estudantes, com idades entre 13 a 20 anos $(M=16,80 ; D P=0,99)$, sendo a maior parte do sexo feminino (54\%), de classe média (45,1\%), do terceiro ano (54,9\%). Esses responderam os seguintes instrumentos: FCBVSs, Escala de Comportamentos de Bullying (ECB) e um Questionário Sociodemográfico. A Análise Fatorial Confirmatória apontou o modelo com três fatores como o mais adequado, tendo os três fatores correspondentes Alfas de Cronbach e Ômegas de McDonald superiores a 0,70. Desse modo, verifica-se que a FCBVSs se apresenta como uma medida válida e fidedigna, podendo ser utilizada em estudos futuros para avaliar o cyberbullying e os seus correlatos.
\end{abstract}

Palavras-chave: bullying virtual, vitimização, validade, precisão.

\section{Psychometric parameters of the Florence Scale Cyberbullying-Cybervitimization}

\begin{abstract}
The aim of this study was to adapt CyberVictimization Cybernetics (FCBVSs) to the Brazilian context, combining fatal evidence and internal consistency, as well as the invariable factorial by the measure in relation to the participants' sex. There were 315 students aged 13 to 20 years (M $=16.80, \mathrm{SD}=0,99)$, the majority of whom were female $(54 \%)$, middle class $(45.1 \%)$, of the third year $(54.9 \%)$. These responded to (FCBVSs), Bullying Behavior Scale (ECB) and the sociodemographic Questionnaire. The factorial confirming the modeling with more parameters as
\end{abstract}


the most appropriate, plus the corresponding references of Cronbach and McDonald gamma masses higher than 0.70. In this way, it verifies if a FCBVSs presents a valid and reliable measurement, and can be used in future studies to know the correlates of cyberbullying.

Keywords: virtual bullying, victimization, validy, precision.

\section{Parámetros psicométricos de la Escala Florencia Cyberbullying-Cybervitimização}

\section{Resumen}

El presente estudio objetivó adaptar la Escala Florence Cyberbullying-Cybervitimización (FCBVSs) para el contexto brasileño, reuniendo evidencias de validez (factorial y convergente); así como de consistencia interna. Para ello, se contó con una muestra de 315 estudiantes, con edades entre 13 a 20 años $(M=16,80, D P=0,99)$, siendo la mayor parte del sexo femenino (54\%), de clase media (de media) 45,1\%), del tercer año (54,9\%). Estos respondieron los siguientes instrumentos: FCBVSs, Escala de Comportamientos de Bullying (ECB) y un Cuestionario Sociodemográfico. El Análisis Factorial Confirmatorio apuntó el modelo con tres factores como el más adecuado, teniendo los tres factores correspondientes Alfas de Cronbach y Ômegas de McDonald superiores a 0,70. De este modo, se verifica que la FCBVS se presenta como una medida válida y fidedigna, pudiendo ser utilizada en estudios futuros para evaluar el cyberbullying y sus correlatos.

Palabras clave: bullying virtual, victimización, validez, exactitud.

Com o avanço da tecnologia, o bullying, fenômeno que ocorre de forma repetida, intencional, dentro de uma relação desigual de poder (Olweus, 2013; Olweus \& Limber, 2018), vem ultrapassando as paredes da escola, dando origem ao cyberbullying. Esse tipo de intimidação tem sido apontado como um problema que ameaça a saúde pública de jovens e adolescentes, dadas as consequências que o mesmo pode acarretar ao bem-estar e à saúde biopsicossocial dos envolvidos (Bergmann \& Baier, 2018; Chen, Ho, \& Lwin, 2017; Kowalski, Giumetti, Schroeder, \& Lattanner, 2014; Matos, Vieira, Amado, Pessoa, \& Martins, 2018; Schneider, O’Donnell, Stueve, \& Coulter, 2012; Wendt \& Lisboa, 2014).

Nesse sentido, o cyberbullying emerge como uma extensão do bullying, podendo ser definido como um tipo de maltrato ou violência entre pares, através da internet e dispositivos móveis, que visa denegrir, humilhar, caluniar, ameaçar e fazer chantagens com os seus pares (Avilés, 2013; Nocentini et al., 2010; Olweus \& Limber, 2018; Slonje \& Smith, 2008; Smith, Steffgen, \& Sittichai, 2013; Tognetta \& Bozza, 2012). Para Palladino, Nocentini e Menesini (2015), o cyberbullying é um fenômeno multidimensional, podendo ser classificado segundo a natureza do ataque, a saber: comportamentos de escrita verbal (por exemplo, telefonemas, mensagens de texto e e-mails maldosos); comportamentos visuais (por exemplo, postar fotos e vídeos que comprometam a imagem do outro); comportamentos de falsificação (por exemplo, fazer uso de informações pessoais de outra pessoa, como senhas de redes sociais, para enviar mensagens ou outro tipo de comunicação); e comportamentos de exclusão (por exemplo, excluir intencionalmente alguém de um grupo). Para esses autores, o indivíduo pode se envolver nesses comportamentos tanto no papel de vítima (Cyber Vitimização) a qual diz respeito aos alvos da vitimização; quanto ao papel de agressor (Cyber Agressão) que se refere àqueles que cometem a agressão.

Vale ressaltar ainda que dois aspectos são descritos na literatura como essenciais na distinção entre o cyberbullying e a intimidação presencial, são esses: (1) o anonimato, que implica na impressão da ausência de consequências para os agressores, pois, dificilmente eles serão identificados; e (2) a audiência, que corresponde a rápida difusão da informação pelas redes sociais (Raskauskas \& Stoltz, 2007; Smith et al., 2008; Tognetta \& Bozza, 2012). Diante disso, o cyberbullying pode apresentar implicações tão graves quanto o bullying tradicional, já que as ofensas contra uma pessoa, espalhadas rapidamente, acabam condenando-a ao isola- 
mento social, provocando exclusão do seu grupo social por meio das fofocas, da difamação e de boatos.

A despeito disso, o estudo de revisão proposto por Kowalski et al. (2014) aponta que vítimas de cyberbullying tendem a relatar altos níveis de estresse $(\mathrm{r}=0,34)$, ideação suicida $(\mathrm{r}=0,27)$, depressão $(\mathrm{r}=0,24)$, ansiedade $(\mathrm{r}=0,24)$, solidão $(\mathrm{r}=0,24)$, sintomas somáticos $(\mathrm{r}=0,19)$, problemas emocionais $(\mathrm{r}=0,18)$, uso de drogas e álcool $(\mathrm{r}=0,15)$, bem como, apresentam uma redução na satisfação com a vida $(r=-0,21)$, na autoestima $(r=-0,17)$, e nos comportamentos pró-sociais $(\mathrm{r}=-0,05)$. Não apenas as vítimas, mas os agressores também sofrem consequências dessa agressão, de modo que ser um perpetrador de $\mathrm{CB}$ pode estar associado ao uso de drogas e álcool ( $\mathrm{r}=$ $0,27)$, ansiedade $(\mathrm{r}=0,16)$ e depressão $(\mathrm{r}=0,15)$. Além disso, perpetradores do CB tendem a ser mais propensos a relatar baixa satisfação com a vida $(r=-0,11)$, baixa autoestima $(r=-0,10)$, e baixo desempenho acadêmico $(\mathrm{r}=-0,09)$, além de níveis mais altos de solidão $(\mathrm{r}=0,09)$.

No que tange à prevalência desse fenômeno, uma revisão de 159 estudos sobre o cyberbullying realizada entre os anos de 2004 a 2014, indicou uma variação de $1,0 \%$ a $61,1 \%$ para vítimas; $3,0 \%$ a $39,0 \%$, para agressores; e 1,5\% a 72,0\%, para ambos papéis (vítimas e agressores; Brochado, Soares, \& Fraga, 2016). Do mesmo modo, estudos recentes de prevalências são verificados em diversos países os quais apontam para taxas entre $1 \%$ a $41 \%$, para o agressor; $3 \%$ a $72 \%$, para vítimas; e 2,3\% a 16,7\% para agressor-vítima (Bergmann \& Baier, 2018; Matos et al., 2018; Selkie, Fales, \& Moreno, 2015).

No Brasil, Wendt (2012) por meio de um estudo com alunos, com idade entre 13 e 17 anos, de escolas públicas e privadas da região metropolitana de Porto Alegre (RS) reportou prevalência de 72,7\% para agressores; 75,6\%, vitimas; e 65,6\%, vítimas-agressoras. Por sua vez, Mallmann, Lisboa e Calza (2018) em seu estudo com adolescentes de 13 a 18 anos, provenientes de escolas localizadas em dois municípios do estado do Rio Grande do Sul, encontraram que mais da metade dos participantes (58\%) estavam envolvidos em cyber- bullying, dos quais, $12,5 \%$ relataram ser vítimas, $10,3 \%$, agressores; e 35,2\%; vítimas-agressoras. Nesse sentido verifica-se uma assimetria de estudo no País, tendo em vista que os estudos existentes se localizam no sul do Brasil, mais especificamente no estado do Rio Grande de Sul, carecendo de estimativas em outros estados para uma melhor confrontação dos resultados.

Face a essas considerações, verifica-se que o tema do cyberbullying se apresenta como relevante, tendo em vista as altas prevalências e graves consequências para os envolvidos. Nesse sentido, com o intuito de melhor compreendê-lo, bem como, conhecer as suas implicações na saúde biopsicossocial dos envolvidos, as pesquisas têm focado na construção de medidas que avaliem o cyberbullying. Acerca disso, em uma revisão internacional, realizada por Berne et al. (2013), foram encontradas 44 medidas disponíveis, não obstante, os autores destacam que nem todas partiram de uma definição preliminar, apresentando um forte dissenso quanto à operacionalização desse construto. Além disso, eles verificaram que a maioria dessas medidas não apresentavam estatísticas detalhadas de propriedades psicométricas, sobretudo de validade de construto.

Por sua vez, no contexto brasileiro, foram encontradas cinco medidas, a saber: Forms of Bullying Scale (Santos, Gouveia, Soares, Cavalcanti, \& Goubeia, 2015); Escala de Comportamentos de Bullying (Medeiros et al., 2015); Olweus Bully Victim Questionnaire (Zottis, 2014); Delaware School Climate Survey-Student (DSCS-S) (Holst, 2015); e Revised Cyberbullying Inventory (Wendt, 2012; Mallmann, et al., 2018). Desses achados, a maioria avalia o cyberbullying enquanto tipologia do bullying (Zottis, 2014; Holst, 2015; Santos et al., 2015; Medeiros et al., 2015), restando apenas a Revised Cyberbullying Inventory (Wendt, 2012; Mallmann et al., 2018) como sendo uma medida específica do cyberbullying, não obstante, parte de uma perspectiva unidimensional do construto.

Nessa perspectiva, o presente estudo objetivou adaptar a Escalas Florence de Cyber Agressão - Cyber Vitimização 
(EFCACV) para o contexto brasileiro, reunindo evidências de validade fatorial e consistência interna. São ainda apresentados resultados de validade convergente com uma medida de bullying e comparações entre os sexos. Nesse sentido partiu-se das seguintes hipóteses: (a) espera-se confirmar a estrutura fatorial com 4 dimensões; (b) espera-se que a EFCACV obtenha resultados satisfatórios em termos de consistência interna; (c) espera-se que a EFCACV demonstre validade convergente com uma medida de bullying; e (d) espera-se que a EFCACV não apresente diferenças entre homens e mulheres.

\section{Método}

\section{Participantes}

A amostra foi composta por 315 estudantes adolescentes, com idades entre 13 e 20 anos $(M=16,80$; $D P=$ $0,99)$, sendo a maior parte do sexo feminino (54\%), de classe média (45,1\%), do terceiro ano (54,9\%). A maioria dos participantes afirmou possuir computador $(68,9 \%)$, celular $(92,1 \%)$ e acesso à internet $(88,6 \%)$.

\section{Instrumentos}

Escalas Florence de Cyber Agressão - Cyber Vitimização Foram elaboradas na Itália por Palladino et al. (2015) e consistem em duas escalas, uma de vitimização e outra de perpetração, ambas compostas por quatro fatores, e juntas contabilizam 28 itens. Desses, três itens correspondem ao fator escrita-verbal; quatro itens, ao fator visual; três, ao fator falsificação; e quatro, ao fator exclusão. Os fatores da medida alcançaram bons índices de consistência interna (Kline, 2013): escrita-verbal (vitimização $\alpha=0,71$; perpetração $\alpha=$ 0,70 ); visual (vitimização $\alpha=0,76$; perpetração $\alpha=0,78$ ), falsificação (vitimização $\alpha=0,63$; perpetração $\alpha=0,77$ ), e exclusão (vitimização $\alpha=0,65$; perpetração $\alpha=0,75$ ).

As escalas têm em vista conhecer com que frequência os participantes experimentaram comportamentos ou eventos específicos durante os últimos três meses e deveriam responder em uma escala de 5 pontos, que variou de 1 = "nunca" a 5 = "várias vezes por semana". Os escores totais possíveis variam de 14 a 70, em que os escores mais altos indicam maior frequência de envolvimento no cyberbullying, seja como vítima, na escala de vitimização, seja como agressor, na escala de perpetração.

Convém destacar que se optou por essa medida, inicialmente, por ela permitir avaliar tanto comportamentos de vitimização, quanto de perpetração; em segundo lugar, por ela considerar o cyberbullying um comportamento distinto do bullying; em terceiro, por levar em consideração aspectos contemporâneos sobre o fenômeno, como o anonimato e a audiência. Além disso, ela destaca-se por considerar diferentes meios eletrônicos (telefone; mensagens de texto, chats, sites, redes sociais, mensagens instantâneas, e-mail), bem como, distintos tipos de comportamento (insultos, exclusão, ameaças, imitação, fotos/vídeo/fotos, solicitação sexual). Finalmente, essas escalas apresentam bons parâmetros psicométricos, tais como: validade de construto, convergente, estabilidade temporal, precisão e invariância fatorial em relação ao sexo (Palladino et al., 2015).

Com a permissão dos autores (Palladino et al.,2015) procedeu-se com a tradução e adaptação da medida, tomando por base o processo sugerido por Hutz, Bandeira e Trentini (2015), de modo que a tradução do inglês (idioma original) para o português foi realizada por três pesquisadores psicólogos bilíngues que possuíam o domínio fluente do inglês e conheciam em detalhe as escalas. Posteriormente, foram comparados os textos traduzidos, realizando uma segunda uniformização das escalas ajustando expressões que poderiam gerar dúvidas. Após esse procedimento, a versão da escala foi aplicada em um estudo piloto com 20 alunos universitários, a fim de aferir a compreensão da tradução das escalas. Na ocasião, era solicitado que cada aluno apontasse as possíveis dúvidas ou dificuldades. Após essa etapa, algumas expressões foram modificadas e deu-se prosseguimento para a aplicação da versão final das escalas.

Escala de Comportamentos de Bullying (ECB). Elaborada por Medeiros et al. (2015) é composta por 
16 itens referentes à frequência de comportamentos de bullying na última semana, os quais estão distribuídos igualmente entre quatro fatores distintos. Os fatores abordados, Bullying verbal, Cyberbullying, Bullying físico e Bullying relacional, apresentaram Alfas de Cronbach satisfatórios que variaram de 0,60 a 0,79 (Kline, 2013). O questionário fornece escores totais possíveis de zero a 64 , no qual os escores mais altos indicam maior frequência de comportamentos de bullying.

Questionário sociodemográfico. Também foi utilizado um questionário sociodemográfico com o fim de caracterizar questões sobre como: idade, sexo, série, uso de computador, internet e celular.

\section{Procedimentos}

A coleta de dados nas escolas se deu da seguinte forma: inicialmente obteve-se a permissão do diretor da escola. Após o seu consentimento, foram escolhidas as turmas pelos pesquisadores, mediante a disponibilidade dos professores e do quadro de aulas. A aplicação foi efetuada em ambiente coletivo de sala de aula. Antes de iniciar a coleta os pesquisadores apresentaram o objetivo do estudo aos adolescentes, instruindo-os a responderem ao questionário individualmente, previamente já assinado pelos pais no Termo de Consentimento Livre e Esclarecido e assinado pelo adolescente no termo de assentimento. O tempo médio para que os adolescentes respondessem foi de, aproximadamente, 15 minutos.

Convém destacar que todos os procedimentos empregados no presente estudo foram aprovados pelo Comitê de Ética em Pesquisa da Universidade Federal da Paraíba (UFPB) (Prot. n. ${ }^{\text {o } 0465 / 16 ; ~ C A A E: ~ 58471616.1 .0000 .5188), ~}$ e seguidas as normas da resolução 466/2012 e 510/2016 do Comitê de Ética em Pesquisa com Seres Humanos.

\section{Análise de dados}

Para a análise fatorial confirmatória (AFC), a partir da linguagem $\mathrm{R}$, especificadamente o pacote Lavaan (Rosseel, 2012), empregou-se o método de estimação Weighted Least Squares Mean - and Variance-adjusted
(WLSMV) pois essa estimativa apresenta-se pertinente para todos os tamanhos amostrais (Beauducel \& Herzberg, 2006). Para avaliar a adequação do modelo foram utilizados os seguintes índices de ajuste: o Comparative Fit Index (CFI), o Tukey-Lewis Index (TLI), a Root Mean Square Error of Approximation (RMSEA) (Brown, 2006) e o Standardized Root Mean Square Residual (SRMR) (Byrne, 2010). O CFI e o TLI avaliam o ajuste do modelo comparado com um modelo nulo, e variam de zero a 1, de modo que, valores acima de 0,90 são considerados aceitáveis (Hair, Black, Babin, Anderson, \& Tatham, 2009). A RMSEA é um índice que avalia a parcimônia do modelo em relação ao número de coeficientes estimados, valores abaixo de 0,06 são considerados adequados (Brown, 2006). O índice SRMR compara a matriz dos dados amostrais com um modelo saturado (indicados valores $<0,05$; Brown, 2006).

Além disso, foram calculados os índices de precisão (Alfas de Cronbach e Ômegas de McDonald) através da linguagem $\mathrm{R}$, considerando os resultados apontados para escalas ordinais, superiores ou iguais a 0,70 . Também foram calculadas estatísticas descritivas e inferenciais, como: média, desvio padrão, correlações de Pearson, e test t para amostra independentes, todas pelo IBM SPSS (versão 21).

\section{Resultados}

Partindo do modelo proposto por Palladino et al. (2015) buscou-se verificar a adequação de um modelo de quatro fatores, por meio de análises fatoriais confirmatórias com o estimador WLSMV. Os indicadores de ajustes encontrados para a escala de Cyber Vitimização foram: $\chi^{2} / \mathrm{gl}$ $=1,22, \mathrm{CFI}=0,90, \mathrm{TLI}=0,87, \mathrm{SRMR}=0,08$ e RMSEA $=0,02$ $(\mathrm{IC} 90 \%=0,00-0,04)$. Por sua vez, para o modelo para a escala de Cyber Agressão foram encontrados os seguintes índices de ajuste: $\chi^{2} / \mathrm{gl}=1,05, \mathrm{CFI}=0,93, \mathrm{TLI}=0,91$, SRMR $=0,10$ e RMSEA $=0,14($ IC90\% $\%=0,00-0,03)$.

Como alguns indicadores de ajustes apresentaram-se abaixo do esperado (TLI e SRMR), verificaram-se os pesos fatoriais de cada item. Foi observado que todos 
os itens do fator 1 (escrita verbal) e o item 10 referente ao fator falsificação, não apresentaram pesos fatoriais significativos. Nesse sentido, buscou-se testar um modelo trifatorial, sem o fator 1 , bem como, sem o item 10. Além disso, buscou-se comparar com um modelo alternativo (unifatorial). Conforme observado na Tabela 1, os índices de ajustes foram mais satisfatórios para modelo com três fatores tanto para a escala de Cyber Vitimização quanto para a de Cyber Agressão. Esses modelos podem ser melhor visualizados na Tabela 1 .

Esses modelos são representados nas Figuras 1 e 2, com seus respectivos pesos de regressão, todos estatisticamente diferentes de zero, $\mathrm{p}<0,001$.

\section{Tabela 1.}

Índices de Ajuste dos Modelos Testados para as Escalas Florence de Cyber Agressão-Cyber Vitimização

\begin{tabular}{|c|c|c|c|c|c|c|c|}
\hline Modelos & $\chi^{2}$ & GI & $\chi^{2} / \mathrm{gl}$ & CFI & TLI & SRMR & RMSEA (IC90\%) \\
\hline \multicolumn{8}{|l|}{ Vitimização } \\
\hline Modelo unifatorial & 114,253 & 77 & 1,48 & 0,77 & 0,73 & 0,10 & $0,039(\mathrm{IC} 90 \%=0,023-0,054)$ \\
\hline Modelo trifatorial & 15,599 & 24 & 0,64 & 0,93 & 0,90 & 0,05 & $0,034(\mathrm{IC} 90 \%=0,000-0,061)$ \\
\hline Modelo tetrafatorial & 86,837 & 71 & 1,22 & 0,90 & 0,87 & 0,08 & $0,027(\mathrm{IC} 90 \%=0,000-0,044)$ \\
\hline \multicolumn{8}{|l|}{ Perpetração } \\
\hline Modelo unifatorial & 94,634 & 77 & 1,22 & 0,70 & 0,65 & 0,14 & $0,028(\mathrm{IC} 90 \%=0,000-0,045)$ \\
\hline Modelo trifatorial & 15,596 & 24 & 0,64 & 0,97 & 0,96 & 0,09 & $0,016(\mathrm{IC} 90 \%=0,000-0,050)$ \\
\hline Modelo tetrafatorial & 75,131 & 71 & 1,05 & 0,93 & 0,91 & 0,10 & $0,014(\mathrm{IC} 90 \%=0,000-0,037)$ \\
\hline
\end{tabular}

Nota: $\chi^{2}=$ qui quadrado; $\mathrm{Gl}=$ Grau de liberdade; $\mathrm{CFI}=$ Comparative Fit Index; TLI= Tucker Lewis Index; SRMR= Standardized Residual Mean Root; RMSEA= Standardized Root Mean Square Residual; IC= Intervalo de confiança.

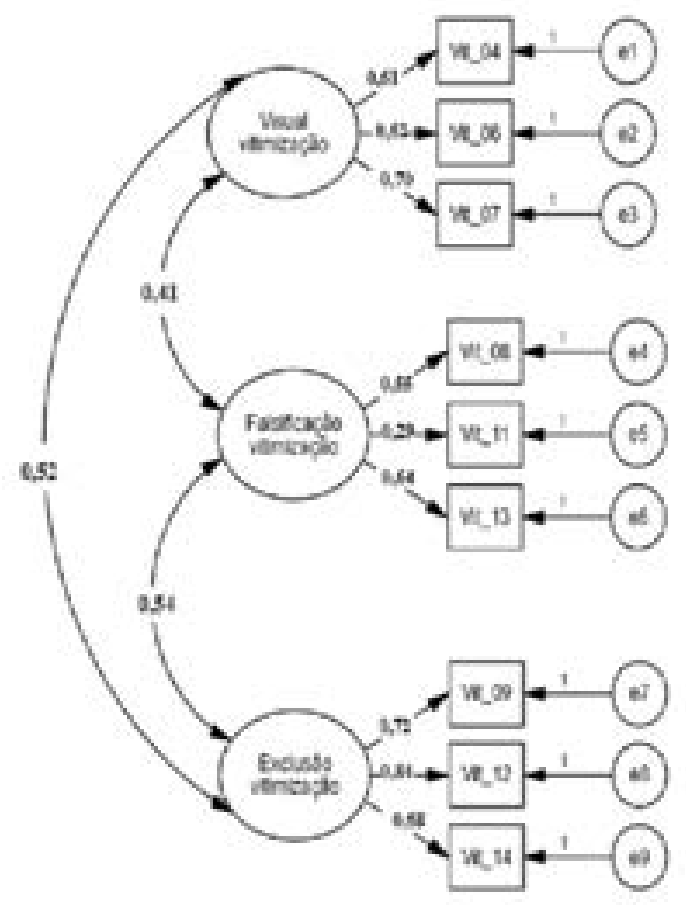

Figura 1. Modelo tri-fatorial de vitimização da Escala de Cyber Vitimização

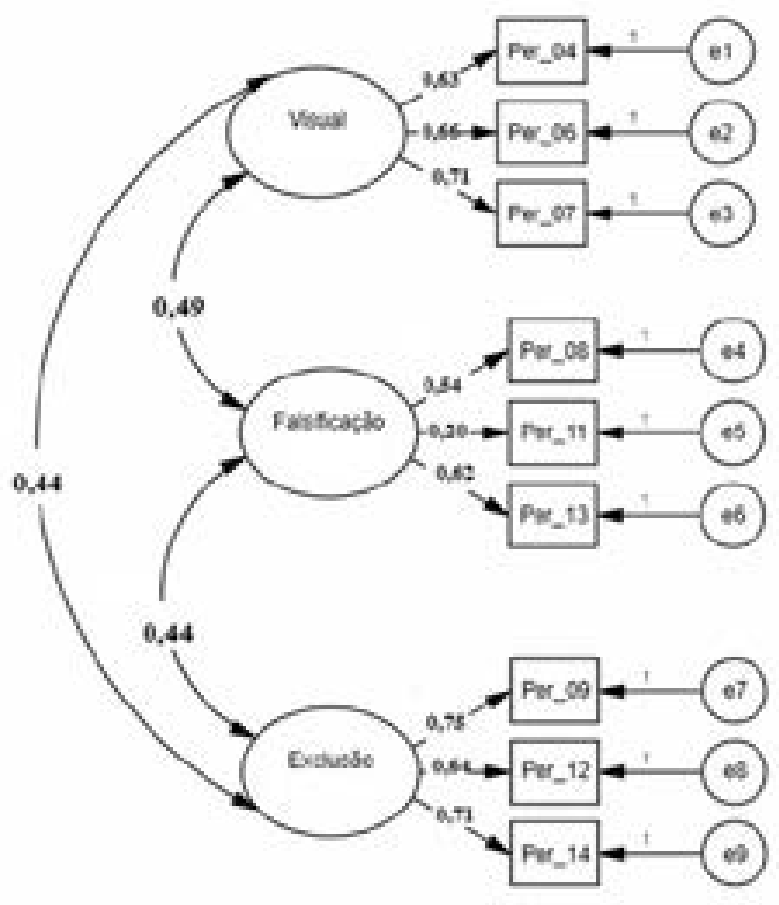

Figura 2. Modelo tri-fatorial de perpetração da Escala de Cyber Agreção 


\section{Consistência interna e validade convergente}

Visando conhecer a consistência interna da medida, foram calculados os Alfas de Cronbach e os Ômegas de McDonald para cada um dos fatores das escalas de Cyber Vitimização e Cyber Agressão. No que tece a escala de Cyber Vitimização, foram encontrados os seguintes índices para os fatores Visual, Falsificação e Exclusão, respectivamente, $\Omega=0,77$ e $\alpha=0,76 ; \Omega=0,80$ e $\alpha=0,80 ; \Omega=0,87$ e $\alpha=0,87$. Quanto a escala de Cyber Agressão, os índices de consistência interna foram: $\Omega=$ 0,$87 ; \alpha=0,86$ (Visual), $\Omega=0,60 ; \alpha=0,60$ (Falsificação); e $\Omega=0,87 ; \alpha=0,84$ (Exclusão).

Além disso, buscou-se verificar a validade convergente das Escalas Florence de Cyber Agressão e Cyber Vitimização com uma escala que mede construtos do bullying. Para escala de Cyber Vitimização, foram encontradas relações positivas do fator visual com o bullying verbal $(\mathrm{r}=0,21 ; \mathrm{p}<0,01)$, físico $(\mathrm{r}=0,10 ; \mathrm{p}<$ $0,05)$, e cyberbullying $(\mathrm{r}=0,14 ; \mathrm{p}<0,05)$. Do mesmo modo, verificou-se uma associação do fator falsificação com o cyberbullying $(\mathrm{r}=0,18 ; \mathrm{p}<0,01)$; e do fator exclusão com bullying verbal $(\mathrm{r}=0,26 ; \mathrm{p}<0,01)$, físico $(\mathrm{r}=0,12 ; \mathrm{p}<0,05)$, e cyberbullying $(\mathrm{r}=0,18 ; \mathrm{p}<0,01)$.

Quando considerada a escala de Cyber Agressão, observaram-se correlações positivas e significativas entre o fator visual e todos os fatores da ECB: bullying verbal $(r=0,36 ; p<0,01)$, físico $(r=0,42 ; p<0,01)$, relacional $(r=0,30 ; p<0.01)$, e cyberbullying $(r=0,10$; $\mathrm{p}<0,05)$. Na mesma direção, verificou-se relações significativas e positivas entre o fator falsificação e todos os fatores da ECB: bullying verbal $(r=0,30 ; p<0,01)$, físico $(r=0,12 ; p<0,01)$, relacional $(r=0,23 ; p<0,01)$, e cyberbullying $(\mathrm{r}=0,19 ; \mathrm{p}<0,05)$. Por fim, o fator exclusão e todos os fatores da ECB, bullying verbal ( $\mathrm{r}$ $=0,53 ; \mathrm{p}<0,01)$, físico $(\mathrm{r}=0,30 ; \mathrm{p}<0,01)$, relacional $(r=0,29 ; p<0,01)$, e cyberbullying $(r=0,20 ; p<0,01)$ correlacionaram-se positivamente. Esses resultados podem ser melhor visualizados na Tabela 2.

\section{Tabela 2}

\section{Correlações entre EFCACV e a ECB}

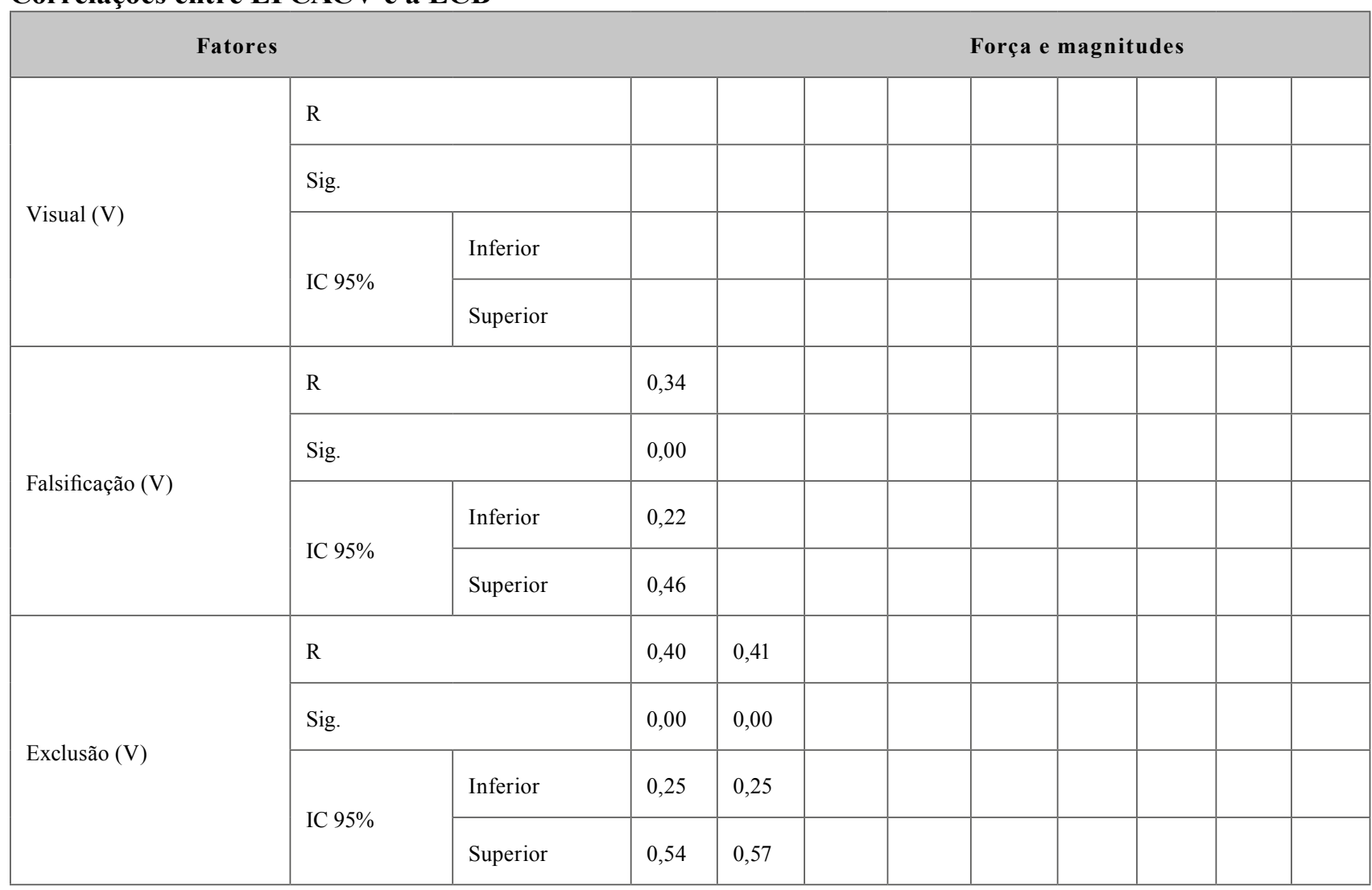




\begin{tabular}{|c|c|c|c|c|c|c|c|c|c|c|c|}
\hline \multirow{4}{*}{ Visual (P) } & \multicolumn{2}{|l|}{$\mathrm{R}$} & 0,31 & 0,06 & 0,03 & & & & & & \\
\hline & \multicolumn{2}{|l|}{ Sig. } & 0,00 & 0,29 & 0,59 & & & & & & \\
\hline & \multirow{2}{*}{ IC 95\% } & Inferior & 0,17 & $-0,03$ & $-0,05$ & & & & & & \\
\hline & & Superior & 0,46 & 0,19 & 0,15 & & & & & & \\
\hline \multirow{4}{*}{ Falsificação (P) } & \multicolumn{2}{|l|}{$\mathrm{R}$} & 0,21 & 0,19 & 0,10 & 0,34 & & & & & \\
\hline & \multicolumn{2}{|l|}{ Sig. } & 0,00 & 0,00 & 0,09 & 0,00 & & & & & \\
\hline & \multirow{2}{*}{ IC $95 \%$} & Inferior & 0,06 & 0,08 & $-0,02$ & 0,12 & & & & & \\
\hline & & Superior & 0,40 & 0,37 & 0,29 & 0,57 & & & & & \\
\hline \multirow{4}{*}{ Exclusão (P) } & \multicolumn{2}{|l|}{$\mathrm{R}$} & 0,35 & 0,25 & 0,40 & 0,35 & 0,40 & & & & \\
\hline & \multicolumn{2}{|l|}{ Sig. } & 0,00 & 0,00 & 0,00 & 0,00 & 0,00 & & & & \\
\hline & & Inferior & 0,20 & 0,12 & 0,24 & 0,16 & 0,24 & & & & \\
\hline & & Superior & 0,49 & 0,40 & 0,55 & 0,51 & 0,53 & & & & \\
\hline \multirow{4}{*}{ Bul_fisico } & \multicolumn{2}{|l|}{$\mathrm{R}$} & 0,11 & 0,05 & 0,10 & 0,29 & 0,13 & 0,20 & & & \\
\hline & \multicolumn{2}{|l|}{ Sig. } & 0,04 & 0,33 & 0,09 & 0,00 & 0,02 & 0,00 & & & \\
\hline & & Inferior & $-0,01$ & $-0,05$ & $-0,00$ & 0,07 & $-0,02$ & 0,08 & & & \\
\hline & & Superior & 0,25 & 0,17 & 0,22 & 0,48 & 0,36 & 0,33 & & & \\
\hline \multirow{4}{*}{ Bul_verbal } & \multicolumn{2}{|l|}{$\mathrm{R}$} & 0,20 & 0,08 & 0,26 & 0,37 & 0,27 & 0,50 & 0,36 & & \\
\hline & \multicolumn{2}{|l|}{ Sig. } & 0,00 & 0,15 & 0,00 & 0,00 & 0,00 & 0,00 & 0,00 & & \\
\hline & & Inferior & 0,08 & $-0,02$ & 0,14 & 0,22 & 0,14 & 0,39 & 0,20 & & \\
\hline & & Superior & 0,32 & 0,21 & 0,37 & 0,50 & 0,42 & 0,60 & 0,50 & & \\
\hline \multirow{4}{*}{ Bul_relacional } & \multicolumn{2}{|l|}{$\mathrm{R}$} & 0,08 & 0,09 & 0,06 & 0,33 & 0,20 & 0,25 & 0,44 & 0,45 & \\
\hline & \multicolumn{2}{|l|}{ Sig. } & 0,17 & 0,12 & 0,24 & 0,00 & 0,00 & 0,00 & 0,00 & 0,00 & \\
\hline & & Inferior & $-0,02$ & $-0,01$ & $-0,04$ & 0,11 & 0,01 & 0,13 & 0,18 & 0,33 & \\
\hline & & Superior & 0,19 & 0,21 & 0,20 & 0,54 & 0,42 & 0,36 & 0,65 & 0,55 & \\
\hline \multirow{5}{*}{ Bul_cyber } & \multicolumn{2}{|l|}{$\mathrm{R}$} & 0,15 & 0,17 & 0,16 & 0,11 & 0,20 & 0,18 & 0,37 & 0,29 & 0,36 \\
\hline & \multicolumn{2}{|l|}{ Sig. } & 0,00 & 0,00 & 0,00 & 0,05 & 0,00 & 0,00 & 0,00 & 0,00 & 0,00 \\
\hline & \multirow{2}{*}{ IC 95\% } & Inferior & $-0,00$ & 0,03 & 0,02 & $-0,02$ & $-0,02$ & 0,05 & 0,14 & 0,15 & 0,13 \\
\hline & & Superior & 0,30 & 0,33 & 0,33 & 0,29 & 0,43 & 0,31 & 0,58 & 0,42 & 0,60 \\
\hline & & & 1 & 2 & 3 & 4 & 5 & 6 & 7 & 8 & 9 \\
\hline
\end{tabular}


Frequência de Cyber Agressão e Cyber Vitimização e diferenças em função do sexo

Finalmente, tomando por base a estrutura com três fatores, procurou-se conhecer o percentual de envolvimento no Cyber Agressão e no Cyber Vitimização, apontando as médias e desvios padrões de cada fator (ver Tabela 4).
Conforme observado, os dados obtidos na escala de Cyber Agressão revelaram que cerca de $41,2 \%$ dos respondentes relataram ter sofrido em algum momento violência visual (nos últimos 3 meses) sendo a maioria na frequência de 1 a 2 vezes nos últimos 3 meses $(29,2 \%) ; 16,8 \%$ sofreram com a falsificação, sendo a

\section{Tabela 3}

Frequência e médias das Escalas Florence de Cyber Agressão-Cyber Vitimização

\begin{tabular}{|c|c|c|c|c|c|c|}
\hline & $\begin{array}{c}\text { Nunca } \\
\%\end{array}$ & $\begin{array}{c}\text { Apenas } 1 \text { a } 2 \\
\text { vezes } \\
\%\end{array}$ & $\begin{array}{c}2 \text { a } 3 \text { vezes } \\
\text { no mês } \\
\%\end{array}$ & $\begin{array}{c}\text { Uma vez por } \\
\text { semana } \\
\%\end{array}$ & $\begin{array}{c}\text { Várias vezes } \\
\text { por semana } \\
\%\end{array}$ & $\begin{array}{c}\text { Total } \\
\%(\mathrm{M} ; \mathrm{DP})\end{array}$ \\
\hline \multicolumn{7}{|l|}{ Vitimização } \\
\hline Visual & $58,7 \%$ & $29,2 \%$ & $8,3 \%$ & $3,2 \%$ & $0,5 \%$ & $41,2 \%(M=1,63 ; D P=0,76)$ \\
\hline Falsificação & $83,2 \%$ & $15 \%$ & $1,9 \%$ & $0 \%$ & $0 \%$ & $\begin{array}{c}16,9 \% \\
(\mathrm{M}=1,19 ; \mathrm{DP}=0,34)\end{array}$ \\
\hline Exclusão & $63,8 \%$ & $27,6 \%$ & $4,8 \%$ & $2,8 \%$ & $0,9 \%$ & $\begin{array}{c}36,1 \% \\
(\mathrm{M}=1,50 ; \mathrm{DP}=0,75)\end{array}$ \\
\hline \multicolumn{7}{|l|}{ Perpetração } \\
\hline Visual & $90,2 \%$ & $7,6 \%$ & $1,6 \%$ & $0,6 \%$ & $0 \%$ & $\begin{array}{c}9,8 \% \\
(M=1,63 ; D P=0,76)\end{array}$ \\
\hline Falsificação & $93,3 \%$ & $5,8 \%$ & $0,9 \%$ & $0 \%$ & $0 \%$ & $\begin{array}{c}6,7 \% \\
(\mathrm{M}=1,08 ; \mathrm{DP}=0,27)\end{array}$ \\
\hline Exclusão & $58,7 \%$ & $32,1 \%$ & $5,4 \%$ & $2,8 \%$ & $0,9 \%$ & $\begin{array}{c}38,4 \% \\
(\mathrm{M}=1,08 ; \mathrm{DP}=0,27)\end{array}$ \\
\hline
\end{tabular}

maioria de 1 a 2 vezes (15\%); e 36,1\%, exclusão, sendo a maioria de 1 a 2 vezes $(27,6 \%)$. No que concerne a escala de Cyber Vitimização, 9,8\% indicaram ter cometido em algum momento violência visual, sendo a maioria 1 a 2 vezes $(7,6 \%) ; 6,7 \%$ cometeram a falsificação, a maior parte 1 a 2 vezes (5,8\%); e 38,4\%, exclusão, sendo a maioria 1 a 2 vezes $(32,1 \%)$.

Em seguida, buscou-se conhecer em que medida homens e mulheres se diferenciavam em suas pontuações nesses fatores, através do teste t de Student para amostras independentes. A homogeneidade de variância foi testada pelo teste de Levene, a qual atendeu a pressuposição de homogeneidade de variâncias ( $p>0.05)$ para todos os fatores, exceto para o fator falsificação da escala de Cyber Vitimização $(p=0,00)$, ou seja, para esse, não se observaram diferenças significativas entre o grupo homens/mulheres. Sendo assim, foi desconsiderado o resultado do teste $t$ para tal fator, posto ter sido assumida a sua homogeneidade. Vale ressaltar que se procedeu com o test $\mathrm{t}$ (usado para amostras com distribuição normais) por tomar por base o fundamento matemático denominado teorema central do limite que assegura que uma amostra constituída de um $\mathrm{n}$ em torno de 30, as médias amostrais apresentam uma distribuição que tende à distribuição normal, independentemente da forma da distribuição da amostra em si (James, 2004). 


\section{Tabela 4}

Teste de comparação de médias entre os gêneros masculino e feminino $(\mathrm{N}=315)$

\begin{tabular}{|c|c|c|c|c|c|c|}
\hline & $\begin{array}{c}\text { Valor } \\
\text { Mínimo - Máximo }\end{array}$ & Sexos & $\mathbf{M}$ & DP & $t$ & Sig. \\
\hline \multirow{2}{*}{ Visual (P) } & $1-4$ & Masculino & 1,19 & 0,45 & \multirow{2}{*}{1,45} & \multirow{2}{*}{0,14} \\
\hline & & Feminino & 1,12 & 0,34 & & \\
\hline \multirow[t]{2}{*}{ Falsificação(P) } & $1-3$ & Masculino & 1,09 & 0,27 & 0,62 & 0,53 \\
\hline & & Feminino & 1,07 & 0,26 & & \\
\hline \multirow[t]{2}{*}{ Exclusão (P) } & $1-5$ & Masculino & 1,60 & 0,78 & 0,46 & 0,63 \\
\hline & & Feminino & 1,56 & 0,74 & & \\
\hline \multirow[t]{2}{*}{ Visual (V) } & $1-5$ & Masculino & 1,62 & 0,68 & $-0,16$ & 0,86 \\
\hline & & Feminino & 1,63 & 0,81 & & \\
\hline \multirow[t]{2}{*}{ Exclusão (V) } & $1-5$ & Masculino & 1,45 & 0,72 & $-1,05$ & 0,29 \\
\hline & & Feminino & 1,54 & 0,76 & & \\
\hline
\end{tabular}

\section{Discussão}

Considerando o número escasso de estudos acerca do cyberbullying, não sendo encontrado nenhum instrumento, publicado em periódico científico, com referências psicométricas voltadas, especificamente, para o cyberbullying no contexto brasileiro, decidiu-se adaptar a EFCACV, reunindo evidências de validade (fatorial e convergente) e consistência interna, que atenderam aos objetivos propostos pelo presente estudo.

No que concerne à estrutura fatorial do EFCACV, foi utilizada a Análise Fatorial Confirmatória, uma vez ser essa análise a mais adequada para testar modelos com características preestabelecidas, ou que tenham um aporte teórico os norteando (Marôco, 2010). Portanto, foram testados os modelos (uni, tri e tetrafatorial) utilizando-se do estimador WLSMV, por considerá-lo mais robusto, pois utiliza de correlações policóricas que atestam a condição de independência do construto, indicando que quando existem mais de dois itens por construto as correlações devem seguir na mesma direção, sejam elas positivas ou negati- vas, além disso, os itens como ordinais (Bistaffa, 2010). Os resultados apontaram o modelo trifatorial, constituído por 9 itens, como o mais coerente, muito embora reduzido e não confirmando a hipótese de uma estrutura com quatro dimensões, parte da mesma estrutura fatorial proposta pelos autores (Palladino et al., 2015). Convém destacar ainda que se optou pelo modelo de três fatores, não apenas por indicar melhores índices, mas também por tomar por base a indicação de Cheung e Rensvold (2001) que sugere que dentre os modelos possíveis, seja selecionado o mais parcimonioso. Finalmente, não se pode deixar de destacar que embora todo rigor na tradução e retrotradução, conforme procedimentos indicados por Hutz et al. (2015), aspectos concernentes à cultura e à linguagem local podem ter influenciado nas baixas cargas fatoriais do item 10 e dos itens do fator 1. Não obstante, tal exclusão não implicou em danos para as escalas tendo em vista que partiu da mesma estrutura fatorial indicada originalmente.

No que diz respeito à consistência interna, verificam-se índices de alfa de cronbach similares aos 
encontrados pelo estudo original (Palladino et al., 2015), apontando para valores superiores a 0,70 , valor que tem servido de ponto de corte na literatura (Kline, 2013; Nunnally, 1991; Pasquali, 2010). Além disso, o presente estudo relata outro índice de fidedignidade, o Ômega de McDonald, o qual vem sendo avaliado como uma estimativa mais próxima da confiabilidade do que o alfa (Sijtsma, 2009), apontando para valores acima de 0,70. Desse modo, a segunda hipótese foi confirmada.

No que tange à evidência de validade convergente entre a escala de Cyber Vitimização e a escala de comportamentos de bullying, verificaram-se correlações positivas, porém não tão altas, variando entre $r=0,10$ e 0,26 , o que pode ser explicado pelo fato da medida de bullying tratar apenas de perpetração. Embora as correlações possam ser consideradas baixas, os resultados informam que os indivíduos vítimas de cyberbullying tendem a se envolverem em comportamentos de perpetração do bullying. Esses achados apontam para a bidirecionalidade desse tipo de intimidação, demonstrando que os indivíduos podem assumir tanto o papel de vítimas como de agressores, tendo em vista que muitos que sofreram com esse tipo de violência, podem reproduzir tais comportamentos para com seus pares (Barlett \& Gentile, 2012; Gámez-Guadix, Calvete, Orue, \& Las Hayas, 2016; Gámez-Guadix, Gini, \& Calvete, 2015; Kowalski et al., 2014).

Quanto à escala de Cyber Agressão, foram encontradas correlações positivas com a perpetração de comportamentos de bullying para todos os fatores. As correlações variaram de $r=0,10$ (baixa) a $r=0,53$ (alta), indicando que indivíduos perpetradores de bullying também podem ser perpetradores do cyberbullying. Esses achados corroboram estudos prévios que sugerem uma coocorrência desses tipos de intimidações. Essa sobreposição é um dos fatores que fazem com que o cyberbullying seja tratado como um tipo de bullying por um grupo de pesquisadores (Olweus, 2013; Dehue, Bolman, \& Völlink, 2008), enquanto outro, o tratam como um fenômeno separado, porém relacionado ao bullying (Dooley, Pyzallski, \& Cross, 2009; Menesini, Nocentini, \& Calussi, 2011; Y barra, Boyd, Korchmaros, \& Oppenheim, 2012). Tais resultados levam-nos a considerar que a terceira hipótese colocada foi confirmada.

A respeito da frequência e das médias nos comportamentos de Cyber Agressão e Cyber Vitimização entre os adolescentes desse estudo, os índices de vitimização variaram de $16,1 \%$ a $41 \%$; enquanto os de perpetração, variaram de $6,7 \%$ a $38,4 \%$. Esses achados informam taxas relativamente altas, podendo ser justificados pelo crescente uso de tecnologia entre esse grupo etário, tornando mais suscetíveis a esse tipo de agressão. Além disso, verificou-se que a prevalência de comportamentos de Cyber Vitimização foi maior do que o de Cyber Agressão, o que pode ser explicado pelo fato de a vítima constituir um tipo de papel menos reprovável socialmente, em detrimento do agressor. Acerca disso, sugere-se que estudos futuros repliquem esses dados, controlando a variável da desejabilidade social.

Acerca dos comportamentos mais frequentes de Cyber Vitimização, observou-se um maior percentual para o comportamento visual, o que inclui ações como: postar fotos e vídeos que comprometem a imagem do outro. Por sua vez, o percentual de Cyber Agressão foi maior para o comportamento de falsificação, isto é, quando os agressores fazem uso de informações pessoais de outra pessoa para enviar mensagens ou outro tipo de comunicação. Uma possível explicação para o destaque desses dois tipos de violência pode ser pelo fato de terem uma conotação mais virtual; tendo em vista que a exclusão também pode ser manifestada na forma presencial. Além disso, convém ressaltar os resultados encontrados no estudo de Naruskov, Luik, Nocentini, \& Menesini (2012) com adolescentes estonianos, alemães e espanhóis; os quais indicaram o comportamento visual e o de falsificação, como os tipos mais graves e que melhor representam o cyberbullying em comparação com os outros dois tipos (escrita-verbal e exclusão). Nesse sentido, seria importante que pesquisas futuras avaliassem de que forma o cyberbullying e as 
suas formas de manifestações e implicações vêm sendo informados em nossa realidade.

Quando esses percentuais foram comparados em relação ao sexo, verifica-se que não houve diferenças entre homens e mulheres, confirmando-se a quarta hipótese. Esse resultado vai de encontro aos achados de estudos acerca do bullying tradicional, em que atestam tais diferenças, indicando os meninos como os maiores envolvidos no bullying, tanto no papel de agressor, como no de vítima (Cavalcanti, Coutinho, Pinto, Silva, \& Do Bú, 2018; Rech, Halpern, Tedesco, \& Santos, 2013). O que não significaria que meninas não se envolvem em intimidação entre pares, mas assim como afirmam Moura, Cruz e Quevedo (2011), elas, em geral, tendem a apresentar formas mais sutis ou menos visíveis de expressar tal intimidação. Partindo desses achados chama-se atenção para resultados similares quanto ao cyberbullying (Barlett \& Coyne, 2014), em que não se encontram diferenças entre homens e mulheres, o que reafirma o posicionamento de alguns teóricos que avaliam o cyberbullying enquanto um comportamento distinto do bullying, uma vez não pressupor o contato físico entre o agressor e a vítima; apresentando-se assim, como uma forma indireta de agressão. Por essa razão é de se esperar que homens e mulheres não apresentem diferenças, sobretudo pelos achados de que mulheres tendem a se engajar mais fortemente em intimidações mais indiretas e veladas.

Apesar dos resultados supracitados serem relevantes, torna-se imprescindível elencar algumas limitações do presente estudo. A primeira é a de que as amostras foram do tipo não probabilísticas, não podendo ser consideradas como representativas da população brasileira, nem mesmo da paraibana, pois foram constituídas exclusivamente por estudantes do Ensino Médio, devendo-se portanto ponderar as generalizações dos resultados. Além disso, por se tratar de um construto relacionado à violência, $\mathrm{o}$ comportamento pode ter sido recriminado pela sociedade, ou seja, pode ter havido a desejabilidade social, que influencia os participantes a responderem de forma tendenciosa (Stöber, 2001). Tendo em vista tais limitações, sugerem-se que estudos futuros possam superá-las, bem como, possam verificar a estabilidade temporal desta medida (teste-reteste), validade discriminante e preditiva, além de correlacionar com outras variáveis.

Apesar das limitações, os resultados foram similares aos encontrados por Palladino et al. (2015) no que concerne aos fatores visual, falsificação e exclusão, legitimando a classificação do cyberbullying como construto multidimensional em seus padrões de comportamento. Confirmando, portanto, uma boa medida no rastreamento de vítimas e agressores do cyberbullying, que pode servir como um guia para profissionais na elaboração de intervenções eficazes para minimização desse problema. Nesse sentido, intervenções podem ser levadas a cabo, pautadas no empoderamento das vítimas, encorajando-as a denunciar ações de cyberbullying; no fortalecimento dos recursos subjetivos (habilidades sociais, assertividade, empatia) dos adolescentes para enfrentamento do problema; e na educação de pais acerca dos potenciais problemas do mau uso da tecnologia.

\section{Referências}

Avilés, J. M. (2013). Análisis psicosocial del ciberbullying: claves para una educación moral. Papeles del Psicólogo, 34(1), 65-73. (DOI INEXISTENTE)

Barlett, C. P., \& Gentile, D. A. (2012). Attacking others online: The formation of cyberbullying in late adolescence. Psychology of Popular Media Culture, 1(2), 123-135. doi: 10.1037/a0028113.

Barlett, C., \& Coyne, S. M. (2014). A Meta Analysis of Sex Differences in Cyber Bullying Behavior: The Moderating Role of Age. Aggressive Behavior, 40, 474488. doi: 10.1002/ab.21555

Beauducel, A., \& Herzberg, P. Y. (2006) On the Performance of Maximum Likelihood Versus Means and Variance Adjusted Weighted Least Squares Estimation in CFA, Structural Equation Modeling: A Multidisciplinary Journal, 13:2, 186-203, doi: 10.1207/ s15328007sem1302_2

Bergmann, M. C., \& Baier, D. (2018). Prevalence and correlates of cyberbullying perpetration. Findings from 
a German representative student survey. International Journal of Environmental Research and Public Health, 15(2), 274. https://doi.org/10.3390/ijerph15020274

Berne, S., Frisén, A., Schultze-Krumbholz, A., Scheithauer, H., Naruskov, K., Luik, P. ... \& Zukauskiene, R. (2013). Cyberbullying assessment instruments: A systematic review. Aggression and Violent Behavior, 18(2), 320-334. doi: 10.1016/j.avb.2012.11.022

Bistaffa, B. C. (2010). Incorporação de indicadores categóricos ordinais em modelos de equações estruturais. Dissertação de Mestrado, Universidade de São Paulo, São Paulo, Brasil. https://doi.org/10.11606/d.45.2010. tde-09022011-110229

Brochado, S., Soares, S., \& Fraga, S. (2016). A scoping review on studies of cyberbullying prevalence among adolescents. Trauma, Violence, \& Abuse, 18(5), 523-531. doi: $10.1177 / 1524838016641668$.

Brown, T. A. (2006). Confirmatory factor analysis for applied research. New York: Guilford Press.

Byrne, B. M. (2010). Structural equation modeling with Amos: Basic concepts, applications, and programming. New York: Routledge.

Cavalcanti, J. G., de Lima Coutinho, M. D. P., de Lima Pinto, A. V., Silva, K. C., \& Do Bú, E. A. (2018). Vitimização e percepção do bullying: Relação com a sintomatologia depressiva de adolescentes. Revista de Psicologia da IMED, 10(1),140-159.doi: 10.18256/21755027.2018.v10i1.2725

Chen, L., Ho, S. S., \& Lwin, M. O. (2017). A meta-analysis of factors predicting cyberbullying perpetration and victimization: From the social cognitive and media effects approach. New Media \& Society, 19(8), 11941213. doi: $10.1177 / 1461444816634037$.

Cheung, G. W., \& Rensvold, R. B. (2001). The effects of model parsimony and sampling error on the fit of structural equation models. Organizational Research Methods, 4(3), 236-264. doi: 10.1177/109442810143004.

DeHue, F., Bolman, C., \& Völlink, T. (2008). Cyberbullying: Youngsters' experiences and parental perception. CyberPsychology \& Behavior, 11(2), 217223.doi: $10.1089 / \mathrm{cpb} 2007.0008$.

Dooley, J. J., Pyżalski, J., \& Cross, D. (2009). Cyberbullying versus face-to-face bullying: A theoretical and conceptual review. Zeitschrift für
Psychologie/Journal of Psychology, 217(4), 182-188. doi: 10.1027/0044-3409.217.4.182.

Gámez-Guadix, M., Calvete, E., Orue, I., \& Las Hayas, C. (2015). Problematic Internet use and problematic alcohol use from the cognitive-behavioral model: A longitudinal study among adolescents. Addictive Behaviors, 40, 109-114. doi: 10.1016/j.addbeh.2014.09.009

Gámez-Guadix, M., Gini, G., \& Calvete, E. (2015). Stability of cyberbullying victimization among adolescents: Prevalence and association with bully-victim status and psychosocial adjustment. Computers in Human Behavior, 53, 140-148. doi: 10.1016/j.chb.2015.07.007.

Hair, Jr., J. F., Black, W. C., Babin, B. J., Anderson, R. E., \& Tatham, R. L. (2009). Multivariate data analysis. Upper Saddle River, NJ: Pearson Prentice Hall.

Holst, B. (2015). Evidências de validade da escala de clima escolar Delaware School Climate SurveyStudent (DSCS-S) no Brasil. Dissertação de Mestrado. Pontifícia Universidade Católica do Rio Grande do Sul, Porto Alegre, Brasil. https://doi.org/10.24873/j. rpemd.2018.06.219

Hutz, C. S., Bandeira, D. R., \& Trentini, C. M. (2015). Psicometria. Porto Alegre: Artmed Editora.

James, B. R. (2004). Probabilidade: um curso em nivel intermediário. 3a ed. Rio de Janeiro: IMPA.

Kline, P. (2013). Handbook of Psychological Testing. London: Routledge.

Kowalski, R. M., Giumetti, G. W., Schroeder, A. N., \& Lattanner, M. R. (2014). Bullying in the digital age: A critical review and meta-analysis of cyberbullying research among youth. Psychological Bulletin, 140(4), 1073-1137. doi: 10.1037/a0035618.

Mallmann, C. L., de Macedo Lisboa, C. S., \& Calza, T. Z. (2018). Cyberbullying and coping strategies in adolescents from Southern Brazil/Cyberbullying e Estrategias de Coping em Adolescentes do Sul do Brasil/Cyberbullying y estrategias de afrontamiento en adolescents del sur de Brasil. Acta Colombiana de Psicologia, 21(1), 34-44. doi: 10.14718/acp.2018.21.1.2 Marôco, J. (2010). Análise de equações estruturais: Fundamentos teóricos, software \& aplicações. Pêro Pinheiro: Report Number. 
Matos, A. P., Vieira, C. C., Amado, J., Pessoa, T., \& Martins, M. J. D. (2018). Cyberbullying in Portuguese schools: Prevalence and characteristics. Journal of School Violence, 17(1), 123-137. doi: 10.1080/15388220.2016.1263796.

Medeiros, E. D. D., Gouveia, V. V., Monteiro, R. P., Silva, P. G. N. D., Lopes, B. D. J., Medeiros, P. C. B. D., \& Silva, É. S. D. (2015). Bullying Behaviors Scale (BBS): Development and Psychometrics Evidences. PsicoUSF, 20(3), 385-397. doi: 10.1590/1413-82712015200302 .

Menesini, E., Nocentini, A., \& Calussi, P. (2011). The measurement of cyberbullying: Dimensional structure and relative item severity and discrimination. Cyberpsychology, Behavior, and Social Networking, 14(5), 267-274. doi: 10.1089/cyber.2010.0002.

Moura, D. R. D., Cruz, A. C. N., \& Quevedo, L. D. Á. (2011). Prevalence and characteristics of school age bullying victims. Jornal de Pediatria, 87(1), 19-23.

Naruskov, K., Luik, P., Nocentini, A., \& Menesini, E. (2012). Estonian students'perception and definition of cyberbullying. Trames: A Journal of the Humanities \& Social Sciences, 16(4), 323-343.doi: 10.3176/tr.2012.4.02

Nocentini, A., Calmaestra, J., Schultze-Krumbholz, A., Scheithauer, H., Ortega, R., \& Menesini, E. (2010). Cyberbullying: Labels, behaviours and definition in three European countries. Journal of Psychologists and Counsellors in Schools, 20(2), 129-142. doi: 10.1375/ ajgc.20.2.129.

Nunnally, J. C. (1970). Introduction to psychological measurement. New York: McGraw-Hill Book Company.

Olweus, D. (2013). School bullying: Development and some important challenges. Annual review of clinical psychology, 9, 751-780. doi: 10.1146/annurev-clinpsy-050212-185516

Olweus, D., \& Limber, S. P. (2018). Some problems with cyberbullying research. Current opinion in psychology, 19, 139-143. doi: 10.1016/j.copsyc.2017.04.012.

Palladino, B. E., Nocentini, A., \& Menesini, E. (2015). Psychometric properties of the Florence cyberbullying-cybervictimization scales. Cyberpsychology, Behavior, and Social Networking, 18(2), 112-119. doi: 10.1089/ cyber.2014.0366

Pasquali, L. (2010). Instrumentação psicológica: Fundamentos e práticas. Porto Alegre: ArtMed.
Raskauskas, J., \& Stoltz, A. D. (2007). Involvement in traditional and electronic bullying among adolescents. Developmental Psychology, 43(3), 564-575. doi: 10.1037/0012-1649.43.3.564.

Rech, R. R., Halpern, R., Tedesco, A., \& Santos, D. F. (2013). Prevalence and characteristics of victims and perpetrators of bullying. Jornal de Pediatria, 89(2), 164-170. https://doi.org/10.1016/j.jpedp.2012.09.004

Rosseel, Y. (2012). Lavaan: An R package for structural equation modeling and more. Version $0.5-12$ (BETA). Journal of statistical software, 48(2), 1-36. (DOI INEXISTENTE)

Santos, L. C. O., Gouveia, R. S., Soares, A. K. S., Cavalcanti, T. M., \& Goubeia, V. V. (2015). Forms of Bullying Scale: Evidências de validade de construto da versão brasileira. Avaliaçao Psicologica: Interamerican Journal of Psychological Assessment, 14(1), 23-31. doi: 10.15689/ap.2015.1401.03.

Schneider, S.K., O’Donnell, L., Stueve, A. \& Coulter, R. W. (2012). Cyberbullying, school bullying, and psychological distress: A regional census of high school students. American Journal Public Health, 102 (1), 171-177. doi: 10.2105/AJPH.2011.300308.

Selkie, E. M., Fales, J. L., \& Moreno, M. A. (2016). Cyberbullying prevalence among US middle and high school-aged adolescents: A systematic review and quality assessment. Journal of Adolescent Health, 58(2), 125-133.doi: 10.1016/ j. jado health. 2015.09.026.

Sijtsma, K. (2009) On the use, the misuse, and the very limited usefulness of Cronbach's alpha. Psychometrika, 74(1), 107-20. doi: 10.1007/S11336-008-9101-0.

Slonje, R., Smith, P. K., \& FriséN, A. (2013). The nature of cyberbullying, and strategies for prevention. Computers in human behavior, 29(1), 26-32. doi: 10.1016/j.chb.2012.05.024.

Smith, P. K., Mahdavi, J., Carvalho, M., Fisher, S., Russell, S., \& Tippett, N. (2008). Cyberbullying: Its nature and impact in secondary school pupils. Journal of Child Psychology and Psychiatry, 49(4), 376-385. doi: 10.1111/j.1469-7610.2007.01846.x.

Smith, P. K., Steffgen, G., \& Sittichai, R. (2013). The nature of cyberbullying, and an international network. In Smith, P. K. \& Steffgen, G. (Eds). Cyberbullying through the new media: Findings 
from an international network, Londres: Psychology

Press. https://doi.org/10.4324/9780203799079

Stöber, J. (2001). The Social Desirability Scale-17 (SDS-

17): Convergent validity, discriminant validity, and relationship with age. European Journal of Psychological Assessment, 17, 222-232. https://doi.org/10.1027//1015$\underline{5759.17 .3 .222}$

Tognetta, L. R. P., \& Bozza, T. C. L. (2012). Cyberbullying: um estudo sobre a incidência do desrespeito no ciberespaço e suas relações com as representações que o adolescentes tem de si. Nuances: Estudos sobre Educação, 23(24), 162-178. doi: 10.14572/ nuances. v23i24.1896

Wendt, G. W. (2012). Cyberbullying em adolescentes brasileiros. Universidade do Vale do Rio dos Sinos, Rio Grande do Sul, Brasil. https://doi.org/10.5327/ z1679443520180200

Wendt, G. W., \& Lisboa, C. (2014). Understanding the cyberbullying phenomenon. Trends in

Psychology, 22(1), 39-54. doi: 10.9788/TP2014.1-04.

Ybarra, M. L., Boyd, D., Korchmaros, J. D., \& Oppenheim, J. K. (2012). Defining and measuring cyberbullying within the larger context of bullying victimization. Journal of Adolescent Health, 51(1), 5358. doi: 10.1016/j.jadohealth.2011.12.031.

Zottis, G. A. H. (2012). Bullying na adolescência: associação entre práticas parentais de disciplina e comportamento agressivo na escola. Dissertação de Mestrado. Universidade Federal do Rio Grande do Sul, Porto Alegre, Brasil. https://doi.org/10.29289/ 259453942018v28s1059

Endereço para correspondência: Jaqueline Gomes Cavalcanti Av. Aragão e Melo, N. 861, Torre, João Pessoa-PB, CEP.: 58040-102. cel. (083)98866-4186; Email: gomes.jaqueline@gmail.com

Recebido em: 26/7/2018.

Aprovado em: 20/2/2019.

Publicado em: xx/xx/2019.

\section{Jaqueline Gomes Cavalcanti}

E-mail: gomes.jaqueline@gmail.com

Titulação Acadêmica: Doutora

Afiliação Institucional: Instituto de Educação

Superior da Paraíba

\section{Tamyres Tomaz Paiva}

E-mail: Tamyres.tomaz@hotmail.com

Titulação Acadêmica: Mestre

Afiliação Institucional: Universidade Federal da Paraíba

\section{Carlos Eduardo Pimentel}

E-mail: kdu1976@gmail.com .

Titulação Acadêmica: Doutor

Afiliação Institucional: Universidade Federal da Paraíba

\section{Adriele Vieira de Lima Pinto}

E-mail: adri.vlp8@gmail.com

Titulação Acadêmica: Mestre

Afiliação Institucional: Universidade Federal da

Paraíba

\section{Giovanna Barroca de Moura}

E-mail: giovannabarroca@gmail.com

Titulação Acadêmica: Mestre

Afiliação Institucional: Universidade Estadual do Vale do Acaraú 